\title{
A 1-V Operated MEMS Variable Optical Attenuator Using Piezoelectric PZT Thin-Film Actuators
}

\author{
Chengkuo Lee, Member, IEEE, Fu-Li Hsiao, Takeshi Kobayashi, Kah How Koh, P. V. Ramana, Wenfeng Xiang, \\ Bin Yang, Chee Wei Tan, and D. Pinjala, Member, IEEE
}

\begin{abstract}
A rotational Si mirror driven by PZT actuators has been investigated for variable optical attenuator (VOA) applications. The PZT actuators deploy 3.1- $\mu \mathrm{m}$ PZT layer. The developed PZT-driven microelectromechanical systems VOA comprising a large $\mathrm{Si}$ reflection mirror integrated with a dual core fiber collimator in 3-D light attenuation arrangement. The curve of the attenuation versus driving voltage shows rather uniform changing rate taking the advantage of the linear relation between the optical angle and the driving voltage. Dynamic range of $40-50 \mathrm{~dB}$ is achieved at 1 and 1.2 dc driving voltages, respectively.
\end{abstract}

Index Terms-Microelectromechanical systems (MEMS), mirror, optical MEMS, piezoelectric actuator, PZT, variable optical attenuators (VOAs).

\section{INTRODUCTION}

$\mathbf{M}$ ICROELECTROMECHANICAL systems (MEMS) technology has been proven as an indispensible technology in making wide range products such as inertial sensors, micromirrors for display and telecommunication, and laboratoryon-a-chip. One of the key factors contributed to the remarkable MEMS commercial success is the development of Si-based micromachining technology and CMOS MEMS [1], because integration of micromechanical parts and CMOS circuits has facilitated mass production of MEMS devices from various aspects, e.g., high production yield, low fabrication cost, available foundry service, etc. In particular, flat micromirror driven by MEMS actuators is the essential component for enabling most applications of optical MEMS. Using thick device layer released from a Si-on-insulator (SOI) substrate as the micromirror plate

Manuscript received January 8, 2009; revised March 30, 2009, April 17, 2009, and May 4, 2009. First published September 9, 2009; current version published October 7, 2009. This work was supported by the National University of Singapore, Singapore, under University Research Fund R-263-000-475-112 and Faculty Research Fund R-263-000-358-112/133.

C. Lee is with the Department of Electrical and Computer Engineering, National University of Singapore, Singapore 117576, Singapore, and also with the Institute of Microelectronics (IME), Agency for Science, Technology and Research (A*STAR), Singapore 117685, Singapore (e-mail: elelc@nus.edu.sg).

F.-L. Hsiao, K. H. Koh, and W. Xiang are with the Department of Electrical and Computer Engineering, National University of Singapore, Singapore 117576, Singapore (e-mail: elehfl@nus.edu.sg; u0509584@nus. edu.sg; elexw@nus.edu.sg).

T. Kobayashi is with the National Institute of Advanced Industrial Science and Technology (AIST), Tsukuba 305-8564, Japan (e-mail: takeshikobayashi@aist.go.jp).

P. V. Ramana, B. Yang, C. W. Tan, and D. Pinjala are with the Institute of Microelectronics (IME), Agency for Science, Technology and Research (A*STAR), Singapore 117685, Singapore (e-mail: pvramana@ ime.a-star.edu.sg; yangb@ime.a-star.edu.sg; tancw@ime.a-star.edu.sg; pinjala @ime.a-star.edu.sg).

Color versions of one or more of the figures in this paper are available online at http://ieeexplore.ieee.org.

Digital Object Identifier 10.1109/JSTQE.2009.2022959 is a well-adopted approach. Since the thick and stiff singlecrystal Si layer enables a large freestanding mirror plate without significant deformation [2]. Besides, the well-established deep reactive ion etching (DRIE) can easily pattern the mirror plate and remove the underneath $\mathrm{Si}$ of an SOI substrate so as to release the mirror plate. However, limited material selection in CMOS MEMS and CMOS-compatible processes restricts the appropriate Si-based actuators to be either electrostatic actuator or electrothermal actuator only [3], [4]. Actuators deploying piezoelectric PZT $\left(\mathrm{PbZr}_{x} \mathrm{Ti}_{1-x} \mathrm{O}_{3}\right)$ films are attractive alternatives to $\mathrm{Si}-$ based actuators, because the piezoelectric actuator shows higher output force and smaller driving voltage due to their higher energy density than the other actuators [5], [6]. Various MEMS devices have been demonstrated by using piezoelectric PZT film actuators. These piezoelectric PZT MEMS devices include atomic force microscope (AFM) cantilevers [7], [8] and cantilever sensors [9], micromirrors [10], [11], ultrasonic proximity sensors [12], accelerometers [13], RF MEMS switches [14], energy harvesters [15], [16], etc. In the optical MEMS field, features of large mirror size, large deflection angle, and smaller driving voltage are strongly demanded by applications like optical scanning display, 2-D optical switch, and variable optical attenuator (VOA).

Yasuda et al. have reported a large elliptical Si mirror $(1 \mathrm{~mm} \times$ $2 \mathrm{~mm}$ ) driven by PZT actuators. This 2-D scanning mirror showed large optical scanning angles, i.e., so-called $4 \theta$, where $\theta$ means the mechanical deflection angle, as large as $23^{\circ}(4.3 \mathrm{kHz}$ for X-scan) by $52^{\circ}$ ( $90.3 \mathrm{~Hz}$ for Y-scan) at low driving voltages of typical 10-20 $\mathrm{V}_{\mathrm{ac}}$ with a $5 \mathrm{~V}_{\mathrm{dc}}$ offset [17]. Recently, Kim et al. have demonstrated a smaller Si mirror $(0.5 \mathrm{~mm} \times 0.5 \mathrm{~mm})$ aiming at 2-D optical switch application. This PZT-actuated mirror achieved static optical deflection angle, i.e., so-called $2 \theta$, of $6.1^{\circ}$ at $16 \mathrm{~V}_{\mathrm{dc}}$ [18]. So far, there is no reported data on the PZTdriven mirror for VOA application to the knowledge of authors. This paper reports data of the first research attempt of MEMS VOA using PZT actuators.

The first MEMS-based VOAs have been demonstrated by two groups from Lucent Technology in 1998. Ford and Walker developed a MEMS VOA using a surface-micromachined SiN suspended membrane with $\lambda / 4$ optical thickness above a Si substrate with a fixed $3 \lambda / 4$ spacing. Voltage applied to electrodes on top of the membrane creates an electrostatic force and pulls the membrane closer to the substrate such that the intensity of reflective light is controlled accordingly. Dynamic range of $25 \mathrm{~dB}$ attenuation is achieved under $50 \mathrm{~V}_{\mathrm{dc}}$ load [19]. Barber et al. developed a MEMS VOA using a surface-micromachined poly-Si microshutter arranged between ends of two fibers and aligned 
along the same axis. This shutter is driven by an electrostatic parallel actuator. The attenuation is determined by percentage of light signals blocked by this shutter regarding to various vertical positions, which are controlled by the actuator. This surface-micromachined in-line-type MEMS VOA can achieve attenuation of dynamic range as high as $50 \mathrm{~dB}$ under $25 \mathrm{~V}$ dc load [20]. Later in 1999, a DRIE-derived shutter type of MEMS VOA from the device layer of an SOI substrate with fiber alignment trenches made the testing, alignment, and assembly of MEMS VOA easier [21]. At a dc voltage of $31 \mathrm{~V}$, a maximum attenuation of slightly over $50 \mathrm{~dB}$ was obtained. This work, done by Prof. de Rooji's group, really opens a window for new research attempts in MEMS VOAs. Since then, various electrostatic actuation mechanisms have been developed for VOA applications based on surface-micromachined structures [22]-[25] and DRIE-derived structures [26]-[35]. Except to the early work done by Ford and Walker, all these aforementioned research efforts are referring to the so-called planar light attenuation scheme, i.e., the light path is parallel to the substrate surface. Besides, the driving voltage of these electrostatic-driven VOAs is in the range of 5-30 $\mathrm{V}_{\mathrm{dc}}$. With the aim of reducing driving voltage, various Si-based thermal actuators have been developed for VOA applications [36]-[39]. Driving dc voltage as low as $3 \mathrm{~V}$ has been reported by using an array of thermalactuator-driven surface-micromachined pop-up mirror [40]. Recently, a large vertical Si mirror $(500 \mu \mathrm{m} \times 1200 \mu \mathrm{m})$ driven by a metal-coil-type electromagnetic actuator has been reported to achieve $40 \mathrm{~dB}$ dynamic range under $0.5 \mathrm{~V}_{\mathrm{dc}}$ load [41].

Briefly speaking, the driving voltage and electrical power consumption are solely dominated by the actuator design in VOA application. More importantly, the stability and repeatability of VOA characteristics are strongly related to design and fabrication quality of actuators and microstructures of VOAs. Maintaining the mirror flatness and reducing the mirror surface roughness are major efforts in optimization of VOA microstructures, in which these factors affect the optical characteristics directly for reflective-type attenuation mechanisms used in VOA applications. In this paper, we report the development work of a novel MEMS VOA driven by PZT actuators.

\section{DESIGN AND FABRICATION}

VOA is commonly adopted as a key component to groom power levels across the wavelength-division multiplexing (WDM) spectrum to minimize crosstalk and maintain the desired SNR ratio. The MEMS VOAs deploy the free-space light attenuation technology, and demonstrate their prevalence advantages over other solutions in terms of device features of wavelength independence, protocol and bit rate independence, etc. Thus, MEMS VOAs can reduce incoming light intensity in an analog control manner regardless the difference of wavelength and protocol. As we highlighted in Section I, achieving lower driving voltage and electrical power consumption is the main research attempt in the development of MEMS actuator for VOAs, while we need to consider the stability and repeatability of VOA characteristics in general. An electrostatically driven titling mir-

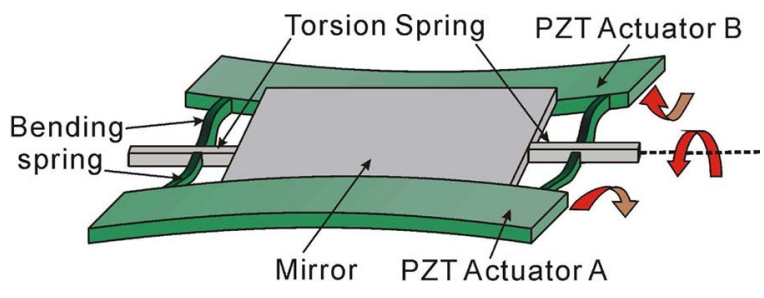

(a)

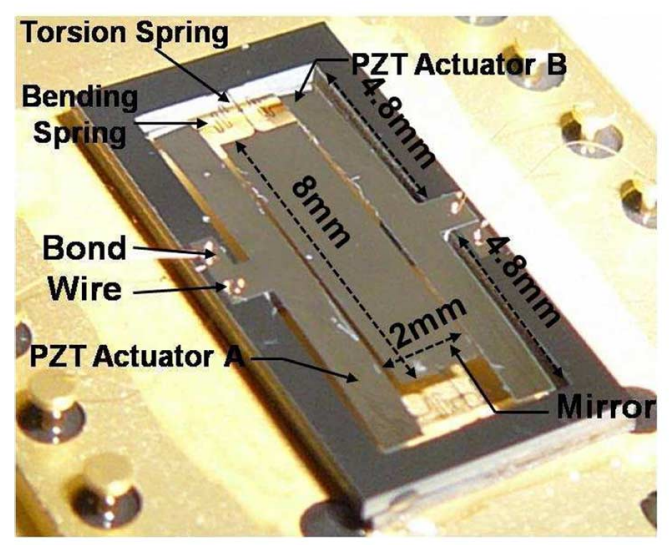

(b)

Fig. 1. (a) Schematic drawing of MEMS VOA. The mirror rotates against the torsion spring due to static displacements introduced by the PZT actuator beams. (b) Optical microscope photograph of a MEMS VOA driven by two piezoelectric PZT actuators.

ror by Toshiyoshi's group has shown $0.3^{\circ}$ rotational angle and 40-dB attenuation at $4.5 \mathrm{~V}_{\mathrm{dc}}$ for VOA applications [42].

A new PZT-driven MEMS VOA is proposed in this paper, as shown in Fig. 1(a), while Fig. 1(b) shows the microfabricated device assembled on a metal case package. A relatively large rectangular mirror $(2 \mathrm{~mm} \times 8 \mathrm{~mm})$ is connected to two piezoelectric $\mathrm{PZT}$ actuators, i.e., actuators $\mathrm{A}$ and $\mathrm{B}$, via bending springs. The size of PZT actuator is $1 \mathrm{~mm} \times 10.6 \mathrm{~mm}$. Torsion springs are arranged along the central line of mirror and connect mirror with peripheral Si substrate. The width of torsional and bending springs, i.e., the width of hinge, is $50 \mu \mathrm{m}$. The structural details of this device are reported by Kobayashi et al. [43]. When the actuators A and B are under reverse dc driving voltage, we can enlarge the static mirror deflection angle by having two piezoelectric actuators bended in opposite directions, as shown in Fig. 1(a).

As shown in Fig. 2(a), an SOI substrate of 5- $\mu$ m-thick Si device layer and 1- $\mu \mathrm{m}$-thick buried oxide (BOX) layer were used as the starting materials. A thermal oxide layer of $0.37 \mu \mathrm{m}$ was created from $\mathrm{Si}$ device layer surface. A multilayer electrode of $\mathrm{LaNiO}_{3}(0.2 \mu \mathrm{m}) / \mathrm{Pt}(0.2 \mu \mathrm{m}) / \mathrm{Ti}(0.05 \mu \mathrm{m})$ was prepared by $\mathrm{RF}$ (for $\mathrm{LaNiO}_{3}$ ) and dc (for Pt/Ti) magnetron sputtering, respectively. Then, a 3.1- $\mu$ m-thick PZT thin film was formed by sol-gel deposition, as reported in our previous study [43]-[45]. The PZT thin film prepared on $\mathrm{LaNiO}_{3}$ buffer layers exhibits columnar structure, as shown in Fig. 3. It has been reported as $(100)$-oriented film [46]. This kind of $(100)$-oriented textured film exhibits higher dielectric constant than the randomly 
(a)

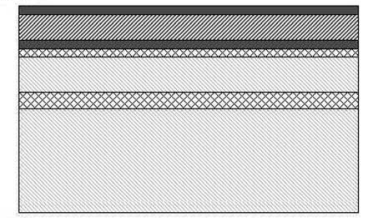

(b)

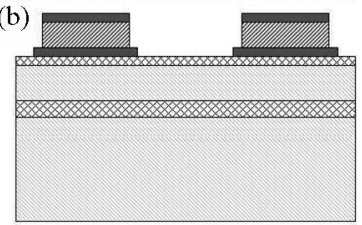

(c)

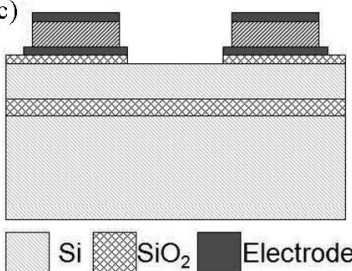

(d)
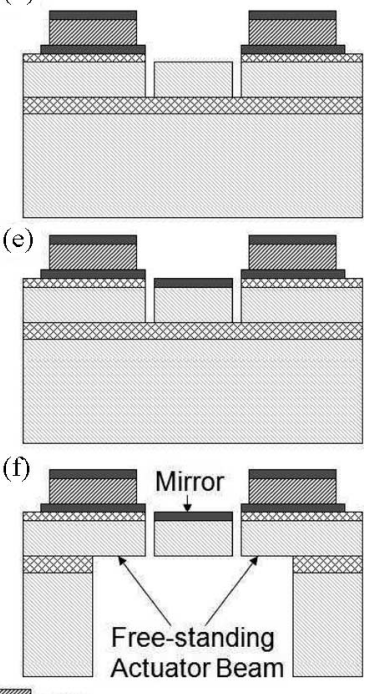

Fig. 2. Microfabrication process flow for making PZT actuators and mirror.

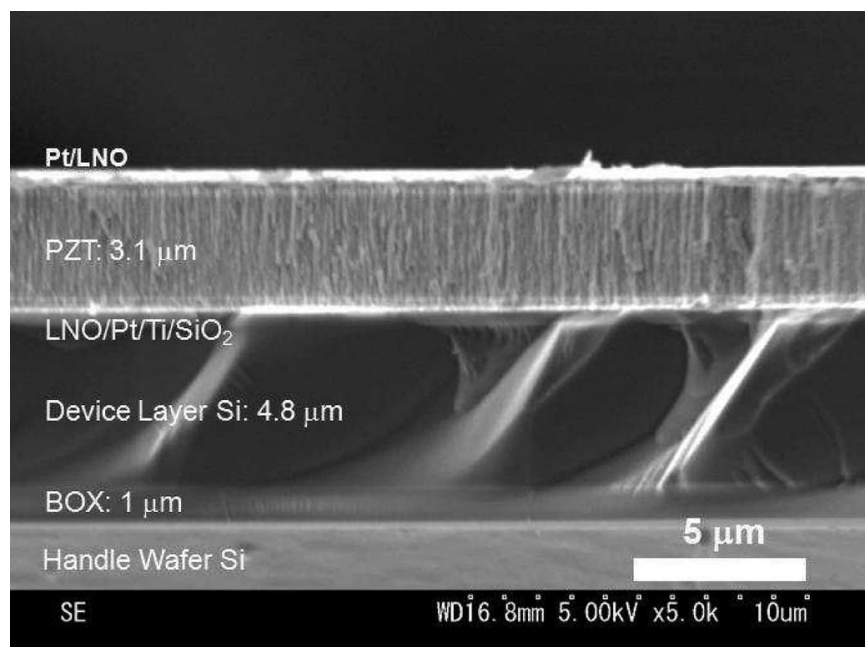

Fig. 3. SEM micrograph of cross-sectional view of PZT actuator thin film on an SOI substrate.

oriented film and the (1 111 )-oriented film [46]. The deposited films were pyrolyzed at $200{ }^{\circ} \mathrm{C}-470{ }^{\circ} \mathrm{C}$ for $5 \mathrm{~min}$, and then, crystallized by rapid thermal annealing at $700{ }^{\circ} \mathrm{C}$ for $2 \mathrm{~min}$. The poling condition is $30 \mathrm{~V}$ for $10 \mathrm{~min}$ at room temperature. The Pt bottom electrode is used because it is a stable metal in this temperature range.

Finally, top electrode of $\mathrm{Pt} / \mathrm{LaNiO}_{3}$ was sputtered on top of the PZT layer. In Fig. 2(b), the top Pt thin film was etched by Ar ion etching and the top $\mathrm{LaNiO}_{3}$ thin film was subsequently etched by diluted $\mathrm{HCl}$. Then, the PZT thin film was etched by the mixture solution of $\mathrm{HF}, \mathrm{HNO}_{3}$, and $\mathrm{HCl}$. Finally, the bottom $\mathrm{LaNiO}_{3} / \mathrm{Pt} / \mathrm{Ti}$ electrode layers were subsequently etched again, while the thermal oxide layer was etched by $\mathrm{CHF}_{3} \mathrm{RIE}$ to open the area of mirror and springs in Fig. 2(c). The mirror, springs, and frame of actuators were patterned from Si device layer of the SOI substrate by DRIE, as shown in Fig. 2(d). Then, a 0.07- $\mu \mathrm{m}-$

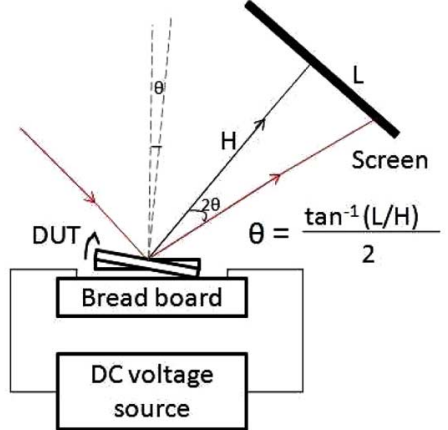

(a)

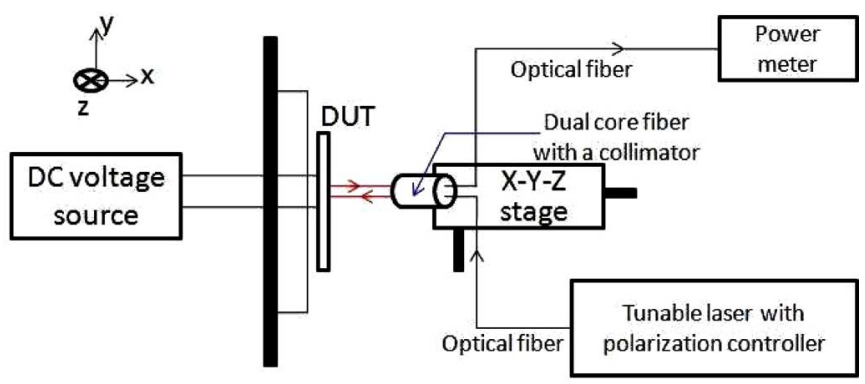

(b)

Fig. 4. (a) Schematic drawing of measurement setup of mirror deflection angle when mirror is driven under dc or ac actuation voltage. (b) Schematic drawing of measurement setup of MEMS VOA characteristics, where the movable stage is capable of moving in $X-Y-Z$ directions and of tilting along $X-Y(\theta z)$ and $X-Z$ $(\theta y)$ planes as well.

thick Pt film was deposited on the mirror as the reflective surface by a liftoff step, as shown in Fig. 2(e). The liftoff method is chosen to avoid the difficulty to find good etching stop materials for $\mathrm{Pt}$ in the Ar ion etching process. We used Pt as the mirror coating layer because we planned to conduct the postprocess annealing for PZT layer initially. Only the Pt is very stable even in the annealing step with temperature as high as $700{ }^{\circ} \mathrm{C}$. Using DRIE, the backside Si portion and BOX were etched from substrate backside to release the mirror, springs, and actuators, as shown in Fig. 2(f), while we used $\mathrm{SF}_{6}$ and $\mathrm{CHF}_{3}$ as the etching feed gas for $\mathrm{Si}$ and $\mathrm{SiO}_{2}$ etching, respectively. To enhance the piezoelectric characteristics, we have conducted the poling treatment of PZT thin-film actuators at $30 \mathrm{~V}$ for $10 \mathrm{~min}$ at room temperature. Fig. 3 shows the SEM photograph of the cross-sectional view of electrodes and PZT actuator layer on top of an SOI substrate. The layer thickness is determined accordingly. Then, we measured the vertical displacement of PZT actuator edge when the PZT actuator was under various dc driving voltages. Based on the approaches discussed in [43]-[45], we derived $d_{31}$ of the present PZT thin films from a set of equations regarding to the displacement changes when the voltage was decreased from 20 to $0 \mathrm{~V}$. The transverse piezoelectric constant $d_{31}$ is estimated as $-110 \mathrm{pm} / \mathrm{V}$. -

\section{Characterization OF PZT MEMS VOA}

The schematic drawing of measurement setup of mirror deflection angle versus driving voltage is shown in Fig. 4(a). The incident light from left side is reflected by mirror and propagates 
toward the screen on right side with optical deflection angle of $2 \theta$, in which $\theta$ means the mechanical deflection angle. When the actuators are driven in ac mode, a mechanical deflection angle $\pm \theta$ is introduced to the mirror. The resultant reflection light will be deviated from the original light path with angle of $\pm 2 \theta$. The light spot on the screen will be shifted with a distance $L$. We can derive the value of $\theta$ from the measured $L$ and the known distance $H$, i.e., the distance from the mirror to the screen.

Fig. 4(b) shows the experimental setup of optical characterization for VOAs. A dual-core fiber with a collimator is placed in the front of the mirror and mounted on the movable $x-y-z$ stages. The input light is launched via one core of the dual-core fiber through collimator to center of the mirror of device. The reflected light is received by the same collimator to power meter via the other core of the dual-core fiber. The collimated beam width is characterized as $750 \mu \mathrm{m} \pm 25 \mu \mathrm{m}$. The large illuminated area reduced the sensitivity of reflection with respect to slightly bending of the mirror. The whole measurement setup is established on top of an antivibration optical table to prevent ambient vibration. We used a tunable laser, which is equipped with a polarization state controller as the light source. The tuning range of light wavelength extended from 1520 to $1620 \mathrm{~nm}$ with $1 \mathrm{~nm}$ resolution. For the initial insertion loss measurement, we need to make sure that the relative positions of collimator and mirror are adjusted to reach the optimized coupling loss, i.e., the minimum coupling loss. In order to do so, we supplied the red laser via collimator and shine to the junction of torsion spring and mirror first. Subsequently, we adjusted the $x-y-z$ stages so as to make the laser spot focused at the mirror center. After we confirmed this coarse alignment step, the 1550-nm laser was fed into the input fiber. The laser light collimated through the collimator and was reflected by the mirror, and then, the reflected light is coupled into the output fiber, which is connected with a laser power meter. In the step of fine alignment, we adjusted the position of collimator by moving and tilting the $x-y-z-\theta y-\theta z$ stages such that we reached minimum insertion loss, i.e., the initial insertion loss. The $\theta y$ and $\theta z$ stages enable the tilting with respect to $y$-and $z$-axis, respectively. The measured insertion loss is typically about $2 \mathrm{~dB}$ in this setup. This initial insertion loss is about $1 \mathrm{~dB}$ higher than commercial products. It is mainly attributed to the surface roughness and warpage of the mirror. Further optimization of the mirror structure and fabrication process may reduce this insertion loss to be less than $1 \mathrm{~dB}$.

Fig. 5 shows the measured optical deflection angle $(2 \theta)$ with respect to ac voltage frequency varying from 50 to $500 \mathrm{~Hz}$, while 2-V peak-to-peak ac voltage $\left(V_{\mathrm{pp}}\right)$ was applied to both actuators $\mathrm{A}$ and $\mathrm{B}$. We made the bottom electrode and top electrode of actuator $\mathrm{A}$ as the ground electrode and driving electrode, respectively. The arrangement is set to be opposite for actuator B. The generated displacements of these two actuators are toward opposite directions such that the deflection angle will be enlarged as the drawing shown in Fig. 1(a). The measured optical deflection angles are $7^{\circ}$ and $3.2^{\circ}$ corresponding to two resonant peaks at 183 and $372 \mathrm{~Hz}$ shown in Fig. 5. Second, Fig. 6 shows the optical deflection angle versus dc driving voltage up to $20 \mathrm{~V}$, while the driving voltage was applied to both actuators A and

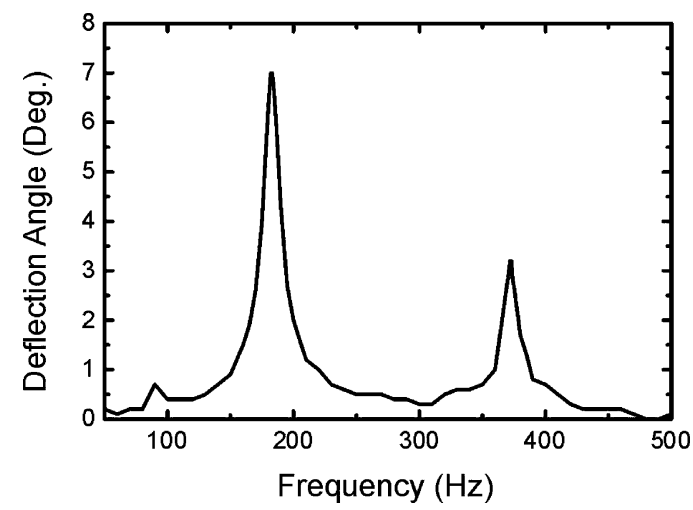

Fig. 5. Optical deflection angle versus ac actuation frequency.

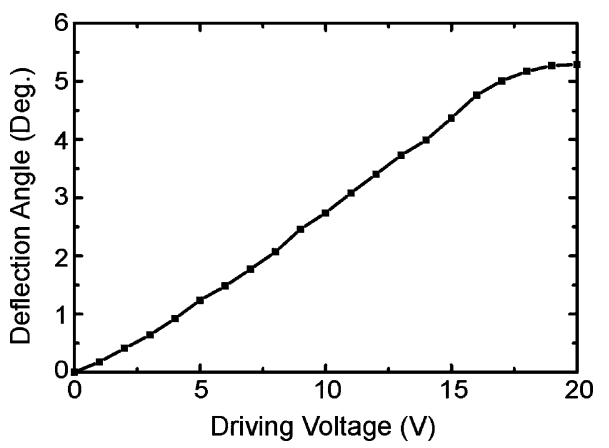

Fig. 6. Measured optical deflection angle versus dc driving voltage.

B under the opposite electrode arrangement. A linear actuation characteristic curve is obtained up to $17 \mathrm{~V}$ with slope of $0.3^{\circ} / \mathrm{V}$. The observed saturation behavior above $17 \mathrm{~V}$ may represent the highest displacement, which can be achieved by the actuators of present configuration.

In the operation of PZT MEMS VOA, we simply apply various dc driving voltages to one or two of the actuators. Then, the reflected light is deviated from the optimized light path corresponding to minimum insertion loss. The insertion loss increases with the driving voltage, i.e., the coupled reflected light intensity toward output fiber is reduced. Based on the VOA characterization setup, as shown in Fig. 4(b), the measured attenuation curves versus dc driving voltage applied to individual actuator are shown in Fig. 7. It shows that $2 \mathrm{~V}_{\mathrm{dc}}$ driving voltage on actuators A and B resulted in 40 and $50 \mathrm{~dB}$ attenuation, respectively. Although the mirror and actuators are arranged in symmetric layout, the laser is very difficult to be aligned at the center of the mirror. This is the main reason that causes the discrepancy of these two measured curves for two actuators. Besides, the dimension of two actuators may not be totally the same due to the lithography inaccuracy and fabrication process variation. Thus, the two PZT actuators may not perform $100 \%$ the same displacement even under the same dc driving voltage. The mirror deflection trace may be slightly twisted from the perfect rotational trace. However, any twist of mirror toward directions other than simple rotation will still lead to attenuation of coupled intensity of reflected light to output port. As a result, the discrepancy of these two measured curves in Fig. 7 is 


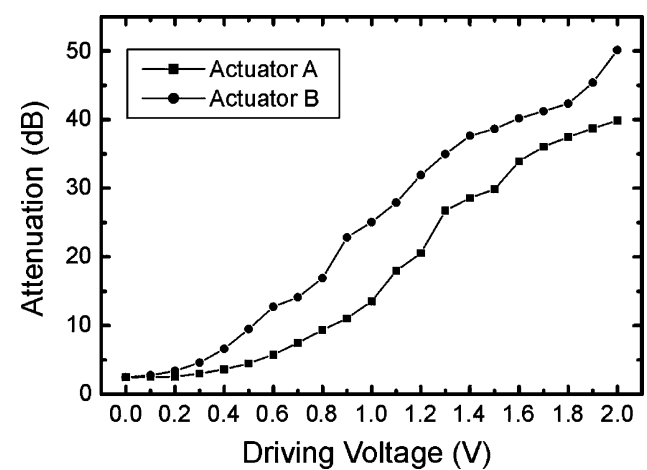

Fig. 7. Measured attenuation curves versus dc driving voltage separately applied to actuators A and B, respectively.

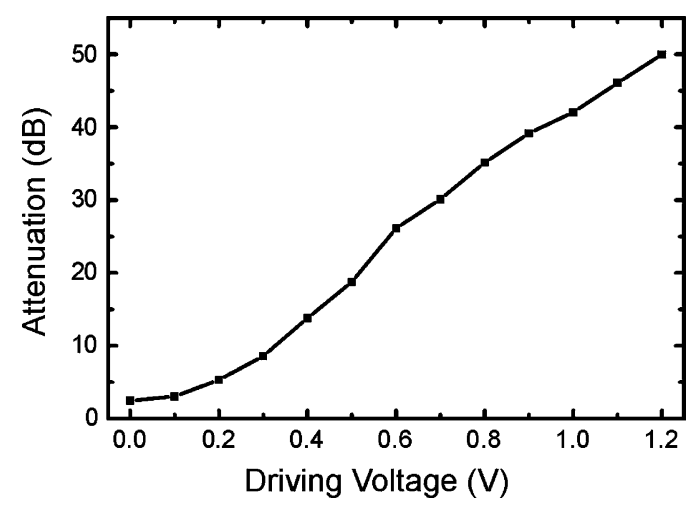

Fig. 8. Attenuation curve when the driving voltage is applied to actuators A and $\mathrm{B}$ at the same time.

partially attributed to the dc-driven displacement difference of two actuators.

Fig. 8 shows the attenuation curve measured when both actuators are driven under the same dc driving voltage. Only $1 \mathrm{~V}_{\mathrm{dc}}$ can achieve $42 \mathrm{~dB}$ dynamic range of attenuation, while $50 \mathrm{~dB}$ is obtained at $1.2 \mathrm{~V}_{\mathrm{dc}}$. The optical deflection angle is measured as $0.18^{\circ}$ at $1 \mathrm{~V}_{\mathrm{dc}}$, as shown in Fig. 6. Referring to most of the commercial applications, $40 \mathrm{~dB}$ dynamic range is enough. It means PZT MEMS VOA developed in this study only requires operation voltage of $1 \mathrm{~V}_{\mathrm{dc}}$. Compared to 3-D MEMS VOA of similar attenuation configuration reported in [42], this 3-D MEMS VOA based on using the electrostatic parallel-plate actuator achieved $40 \mathrm{~dB}$ dynamic range under $5 \mathrm{~V}_{\mathrm{dc}}$ driving voltage in which it is corresponding to mirror angle of $0.3^{\circ}$. In Fig. 8 , the curve of the attenuation versus driving voltage shows rather uniform changing rate taking the advantage of the linear relation between the optical angle and the driving voltage. On the other hand, we applied various driving voltages combination to both actuators and derived an attenuation characteristic topography, as shown in Fig. 9, which shows that we can have one more degree of freedom in attenuation control. More specifically speaking, the deviation of attenuation-driving voltage characteristics among various PZT MEMS VOA devices due to the fabrication process and assembly step could be compensated by changing different driving voltages combination to two actuators.

As shown in Fig. 10, the measured wavelength-dependent loss (WDL) within 1520-1620 nm for driving voltage at $0,0.3$ (10 dB attenuation), 0.5 (20 dB attenuation), and $0.7 \mathrm{~V}(30 \mathrm{~dB}$

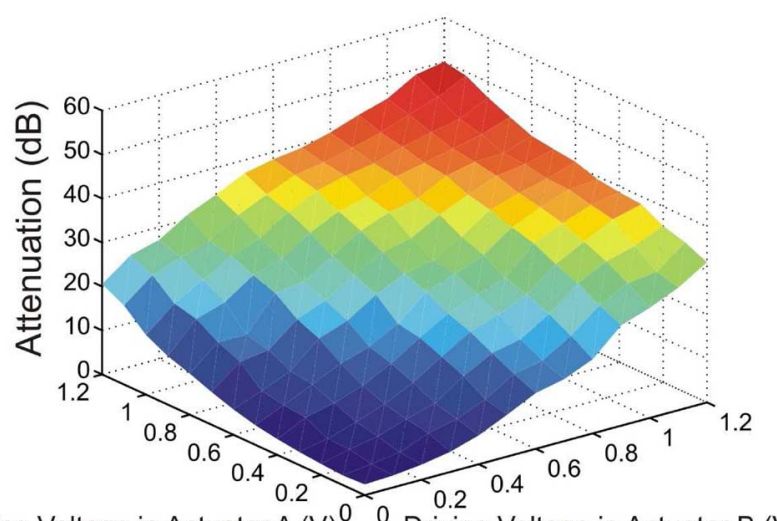

Driving Voltage in Actuator $A(V){ }^{0} 0$ Driving Voltage in Actuator $B(V)$

Fig. 9. Measured attenuation value as a function of driving voltages applied to actuators $\mathrm{A}$ and $\mathrm{B}$.

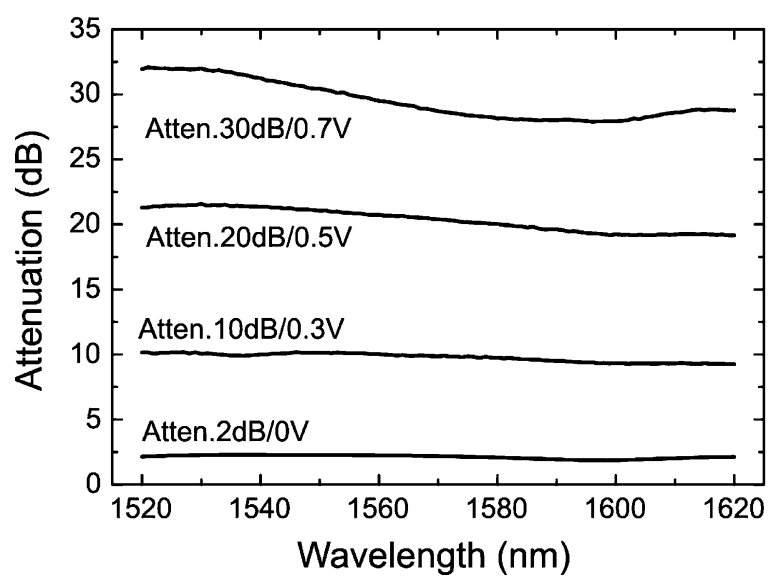

Fig. 10. Measured WDL at various attenuation values.

attenuation) is less than $0.425,0.925,2.418$, and $4.179 \mathrm{~dB}$, respectively. Second, the polarization-dependant loss (PDL) was measured as less than $0.099,0.252,0.271$, and $0.249 \mathrm{~dB}$ at 0,10 , 20 and $30 \mathrm{~dB}$ attenuation states, respectively. Both WDL and PDL data are measured when the same dc driving voltage was applied to both actuators. The WDL data measured at 20 and $30 \mathrm{~dB}$ attenuation states are relatively higher than the reported data [21]-[42]. It may be attributed to the surface roughness issue. For example, the micrometer-scale roughness caused by the facet of grains of PZT thin film will lead to difference in reflected light intensity regarding to light of various wavelengths. Additionally, most of the reported PDL is about $0.2-0.3 \mathrm{~dB}$ at 20-dB attenuation state [21]-[42]. Thus, the derived PDL in this study shows the same level of PDL as previous works reported.

On the other hand, the fatigue issue of PZT thin-film actuator has been investigated by Kobayashi et al. [48]. It reported that a 1.1- $\mu \mathrm{m}$-thick PZT thin-film actuator operated at $1 \mathrm{kHz}$ and $10 \mathrm{~V}$ with superimposed $5 \mathrm{~V}$. The dc-actuated displacement is only reduced for $10 \%$ after $10^{8}$ operation cycles. In this study, the 50-dB attenuation of VOA only demands for $1.2 \mathrm{~V}$, which is calculated as electric field of $0.39 \mathrm{~V} / \mu \mathrm{m}$, while the electric field used in [48] is calculated as $9.1 \mathrm{~V}_{\mathrm{pp}} / \mu \mathrm{m}$ and $4.5 \mathrm{~V} / \mu \mathrm{m}$. It implies that the operation condition of PZT actuators in VOA application only utilizes small portion of actuation capability 
of actuators. In other words, there is large room left for PZT actuator operation without reaching the materials limitation.

\section{CONCLUSION}

In this study, we first explore the feasibility study of 3-D attenuation mechanism using PZT actuators. Only $1 \mathrm{~V}_{\mathrm{dc}}$ is required to achieve $40 \mathrm{~dB}$ dynamic range. Since the actuator beam structure contains 3.1- $\mu \mathrm{m}$ PZT layer, electrodes, and $4.8-\mu \mathrm{m}$ Si layer. Further reduction of driving voltage can be achieved by reducing the Si mechanical layer thickness. However, the tradeoff is the difficulty in maintaining flatness of the reflection mirror. The measured transverse piezoelectric constant $d_{31}$ of fabricated 3.1- $\mu \mathrm{m}$ PZT layer is $-110 \mathrm{pm} / \mathrm{V}$. It implies that there is not much room for further improvement of piezoelectric characteristics. However, piezoelectric PZT layer could be the sensor material as well. Kobayashi et al. [47] have demonstrated a PZT-driven micromirror scanner integrated with PZT displacement sensors. It points out a research direction that PZT MEMS VOAs could include piezoelectric deformation sensor on top of the attenuation mechanism. Thus, we can use the detected signals from displacement sensor as the feedback control reference. An instrument-level piezoelectric MEMS VOAs with electronic feedback control circuits could be developed in this manner.

\section{REFERENCES}

[1] G. K. Fedder, R. T. Howe, T. J. K. Liu, and E. P. Quévy, "Technologies for cofabricating MEMS and electronics," Proc. IEEE, vol. 96, no. 2, pp. 306-322, Feb. 2008.

[2] H. Schenk, P. Dürr, T. Haase, D. Kunze, U. Sobe, H. Lakner, and H. Kück, "Large deflection micromechanical scanning mirrors for linear scans and pattern generation," IEEE J. Sel. Top. Quantum Electron., vol. 6, no. 5, pp. 715-722, Sep./Oct. 2000.

[3] H. Xie, Y. Pan, and G. K. Fedder, "A CMOS-MEMS mirror with curledhinge comb drives," J. Microelectromech. Syst., vol. 12, no. 4, pp. 450457, Aug. 2003.

[4] A. Jain, H. Qu, S. Todd, and H. Xie, "A thermal bimorph micromirror with large bi-directional and vertical actuation," Sens. Actuators A, vol. 122, pp. 9-15, 2005.

[5] R. Maeda, J. J. Tsaur, S. H. Lee, and M. Ichiki, "Piezoelectric microactuator devices," J. Electroceramics, vol. 12, pp. 89-100, 2004.

[6] J. Baborowski, "Microfabrication of piezoelectric MEMS," Integr. Ferroelectr, vol. 66, pp. 3-17, 2004.

[7] C. Lee, T. Itoh, and T. Suga, "Micromachined piezoelectric force sensors based on PZT thin films," IEEE Trans. Ultrason., Ferroelectr., Freq. Control, vol. 43, no. 4, pp. 553-559, Jul. 1996.

[8] C. Lee, T. Itoh, R. Maeda, T. Ohashi, and T. Suga, "Development of a piezoelectric self-excitation and self-detection mechanism of PZT microcantilevers for dynamic SFM in liquid," J. Vac. Sci. Technol. B, vol. 15, pp. 1559-1563, 1997.

[9] S. Shin, N.-E. Lee, H.-D. Park, J.-S. Park, and J. Lee, "Piezoelectrically driven microtransducer mass sensors," Integr. Ferroelectr., vol. 76, pp. $93-$ $100,2005$.

[10] A. Schroth, C. Lee, S. Matsumoto, and R. Maeda, "Application of sol-gel deposited thin PZT film for actuation of 1D and 2D scanners," Sens. Actuators A, vol. 73, pp. 144-152, 1999.

[11] Y. Yee, H.-J. Nam, S.-H. Lee, J. U. Bu, and J.-W. Lee, "PZT actuated micromirror for fine-tracking mechanism of high-density optical data storage," Sens. Actuators A, vol. 89, pp. 166-173, 2001.

[12] K. Yamashita, H. Katata, M. Okuyama, H. Miyoshi, G. Kato, S. Aoyagi, and Y. Suzuki, "Arrayed ultrasonic microsensors with high directivity for in-air use using PZT thin film on silicon diaphragms," Sens. Actuators A, pp. 97-98, 2002

[13] K. Korbinian, P. Enoksson, and G. Stemme, "Highly sensitive triaxial silicon accelerometer with integrated PZT thin film detectors," Sens. Actuators A, vol. 92, pp. 156-160, 2001.
[14] H.-C. Lee, J.-H. Park, and Y.-H. Park, "Development of shunt type ohmic RF MEMS switches actuated by piezoelectric cantilever," Sens. Actuators A, vol. 136, pp. 282-290, 2007.

[15] N. E. duToit, B. L. Wardle, and S.-G. Kim, "Design considerations for MEMS-scale piezoelectric mechanical vibration energy harvesters," Integr. Ferroelectr., vol. 71, pp. 121-160, 2005.

[16] H.-B. Fang, J.-Q Liu, Z.-Y. Xu, L. Dong, L. Wang, D. Chen, B.-C. Cai, and Y. Liu, "Fabrication and performance of MEMS-based piezoelectric power generator for vibration energy harvesting," Microelectron. J., vol. 37, pp. 1280-1284, 2006.

[17] Y. Yasuda, M. Akamatsu, M. Tani, T. Iijima, and H. Toshiyoshi, "Piezoelectric 2D-optical micro scanners with PZT thick films," Integr. Ferroelectr., vol. 76, pp. 81-91, 2005.

[18] S.-J. Kim, Y.-H. Cho, H.-J. Nam, and J. U. Bu, "Piezoelectrically pushed rotational micromirrors using detached PZT actuators for wide-angle optical switch applications," J. Micromech. Microeng., vol. 18, pp. 1250221-125022-7, 2008.

[19] J. E. Ford and J. A. Walker, "Dynamic spectral power equalization using micro-opto-mechanics," IEEE Photon. Technol. Lett., vol. 10, no. 10, pp. 1440-1442, Oct. 1998.

[20] B. Barber, C. R. Giles, V. Askyuk, R. Ruel, L. Stulz, and D. Bishop, "A fiber connectorized MEMS variable optical attenuator," IEEE Photon, vol. 10, no. 9, pp. 1262-1264, Sep. 1998.

[21] C. Marxer, P. Griss, and N. F. de Rooij, "A variable optical attenuator based on silicon micromechanics," IEEE Photon. Technol. Lett., vol. 11, no. 2, pp. 233-235, Feb. 1999.

[22] X. M. Zhang, A. Q. Liu, C. Lu, and D. Y. Tang, "MEMS variable optical attenuator using low driving voltage for DWDM systems," Electron. Lett., vol. 38, no. 8, pp. 382-383, 2002.

[23] A. Q. Liu, X. M. Zhang, C. Lu, F. Wang, C. Lu, and Z. S. Liu, "Optical and mechanical models for a variable optical attenuator using a micromirror drawbridge," J. Micromech. Microeng., vol. 13, pp. 400-411, 2003.

[24] X. M. Zhang, A. Q. Liu, C. Lu, F. Wang, and Z. S. Liu, "Polysilicon micromachined fiber-optical attenuator for DWDM applications," Sens. Actuators A, vol. 108, pp. 28-35, 2003.

[25] C. Lee, Y.-J. Lai, C.-Y. Wu, Y.-S. Lin, M. H. Tsai, R.-S. Huang, and M.-S. Lin, "Scratch drive actuator driven self-assembled variable optical attenuator," Jpn. J. Appl. Phys., vol. 43, pp. 3906-3909, 2004.

[26] C. Chen, C. Lee, and Y.-J. Lai, "Novel VOA using in-plane reflective micromirror and off-axis light attenuation," IEEE Commun. Mag., vol. 41, no. 8, pp. S16-S20, Aug. 2003.

[27] Y. Y. Kim, S. S. Yun, C. S. Park, J.-H. Lee, Y. G. Lee, H. K. Lee, S. K. Yoon, and J. S. Kang, "Refractive variable optical attenuator fabricated by silicon deep reactive ion etching," IEEE Photon. Technol. Lett., vol. 16, no. 2, pp. 485-487, Feb. 2004.

[28] A. Bashir, P. Katila, N. Ogier, B. Saadany, and D. A. Khalil, "A MEMSbased VOA with very low PDL," IEEE Photon. Technol. Lett., vol. 16, no. 4, pp. 1047-1049, Apr. 2004.

[29] C. Chen, C. Lee, and J. A. Yeh, "Retro-reflection type MOEMS VOA," IEEE Photon. Technol. Lett., vol. 16, no. 10, pp. 2290-2292, Oct. 2004.

[30] H. Cai, X. M. Zhang, C. Lu, A. Q. Liu, and E. H. Khoo, "Linear MEMS variable optical attenuator using reflective elliptical mirror," IEEE Photon. Technol. Lett., vol. 17, no. 2, pp. 402-404, Feb. 2005.

[31] C. Lee, "Monolithic-integrated 8CH MEMS variable optical attenuators," Sens. Actuators A, vol. 123-124, pp. 596-601, 2005.

[32] C.-H. Kim and Y.-K. Kim, "MEMS variable optical attenuator using translation motion of $45^{\circ}$ tilted vertical mirror," J. Micromech. Microeng., vol. 15, pp. 1466-1475, 2005.

[33] T.-S. Lim, C.-H. Ji, C.-H. Oh, H. Kwon, Y. Yee, and J. U. Bu, "Electrostatic MEMS variable optical attenuator with rotating folded micromirror," IEEE J. Sel. Topics Quantum Electron., vol. 10, no. 3, pp. 558-562, May/Jun. 2004

[34] J. A. Yeh, S.-S. Jiang, and C. Lee, "MOEMS VOA using rotary comb drive actuators," IEEE Photon. Technol. Lett., vol. 18, no. 10, pp. 1170-1172, May 2006.

[35] M. T.-K. Hou, J.-Y. Huang, S.-S. Jiang, and J. A. Yeh, "In-plane rotary comb-drive actuator for a variable optical attenuator," J. Micro/Nanolith. MEMS MOEMS, vol. 7, no. 4, pp. 043015-1-043015-6, 2008.

[36] J. C. Chiou and W. T. Lin, "Variable optical attenuator using a thermal actuator array with dual shutters," Opt. Commun., vol. 237, pp. 341-350, Jan. 2004.

[37] C. Lee, "MOEMS variable optical attenuator with robust design for improved dynamic characteristics," IEEE Photon. Technol. Lett., vol. 18, no. 6, pp. 773-775, Mar. 2006.

[38] C. Lee, "A MEMS VOA using electrothermal actuators," J. Lightw. Technol., vol. 25, no. 2, pp. 490-498, Feb. 2007. 
[39] C. Lee, "Variable optical attenuator using planar light attenuation scheme based on rotational and translational misalignment," Microsyst. Technol., vol. 13, pp. 41-48, 2007.

[40] C. Lee, Y.-S. Lin, Y.-J. Lai, M. H. Tsai, C. Chen, and C.-Y. Wu, “3-V driven pop-up micromirror for reflecting light toward out-of-plane direction for VOA applications," IEEE Photon. Technol. Lett., vol. 16, no. 4, pp. 1044 1046, Apr. 2004.

[41] S. H. Hung, H.-T. Hsieh, and G.-D. J. Su, "An electro-magnetic micromachined actuator monolithically integrated with a vertical shutter for variable optical attenuation," J. Micromech. Microeng., vol. 18, pp. 0750031-075003-8, 2008 .

[42] K. Isamoto, K. Kato, A. Morosawa, C. Chong, H. Fujita, and H. Toshiyoshi, "A 5-Voperated MEMS variable optical attenuator by SOI bulk micromachining," IEEE J. Sel. Topics Quantum Electron., vol. 10, no. 3, pp. 570-578, May/Jun. 2004.

[43] T. Kobayashi, R. Maeda, and T. Itoh, "Low speed piezoelectric optical microscanner actuated by piezoelectric microcantilevers using $\mathrm{LaNiO}_{3}$ buffered $\mathrm{Pb}(\mathrm{Zr}, \mathrm{Ti}) \mathrm{O}_{3}$ thin film," Smart Mater. Struct., vol. 18, pp. 0650081-065008-6, 2009.

[44] T. Kobayashi, M. Ichiki, T. Noguchi, K. Nakamura, and R. Maeda, "Deflection of wafers and cantilevers with Pt/LNO/PZT/LNO/Pt/Ti/SiO 2 multilayered structures," Thin Solid Films, vol. 516, pp. 5272-5276, 2008.

[45] T. Kobayashi, M. Ichiki, R. Kondou, K. Nakamura, and R. Maeda, "Fabrication of piezoelectric microcantilevers using $\mathrm{LaNiO}_{3}$ buffered $\mathrm{Pb}(\mathrm{Zr}, \mathrm{Ti}) \mathrm{O}_{3}$ thin film," J. Micromech. Microeng., vol. 18, pp. 035007-1035007-5, 2008.

[46] T. Kobayashi, M. Ichiki, J. Tsaur, and R. Maeda, "Effect of multi-coating process on the orientation and microstructure of lead zirconate titanate (PZT) thin films derived by chemical solution deposition," Thin Solid Films, vol. 489, pp. 74-78, 2005.

[47] T. Kobayashi, R. Maeda, T. Itoh, and R. Sawada, "Smart optical microscanner with piezoelectric resonator, sensor, and tuner using $\mathrm{Pb}(\mathrm{Zr}, \mathrm{Ti}) \mathrm{O}_{3}$ thin film," Appl. Phys. Lett., vol. 90, pp. 183514-1-183514-3, 2007.

[48] T. Kobayashi, R. Maeda, and T. Itoh, "A fatigue test method for $\mathrm{Pb}(\mathrm{Zr}, \mathrm{Ti}) \mathrm{O}_{3}$ thin films by using MEMS-based self-sensitive piezoelectric microcantilevers," J. Micromech. Microeng., vol. 18, pp. 115007-1115007-6, 2008.

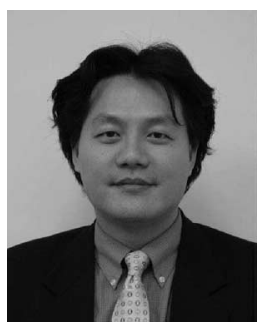

Chengkuo Lee (S'93-M'96) received the M.S. degree in materials science and engineering from the National Tsing Hua University, Hsinchu, Taiwan, in 1991, the M.S. degree in industrial and system engineering from Rutgers University, New Brunswick, $\mathrm{NJ}$, in 1993, and the Ph.D. degree in precision engineering from the University of Tokyo, Tokyo, Japan, in 1996.

From 1993 to 1996, he was a Foreign Researcher in the Nanometer-Scale Manufacturing Science Laboratory, Research Center for Advanced Science and Technology (RCAST), University of Tokyo. During 1996, he was a Japan Science and Technology (JST) Research Fellow in the Mechanical Engineering Laboratory, Advanced Industrial Science and Technology (AIST), Ministry of International Trade and Industry (MITI), Japan. He was a Senior Research Staff in the Microsystems Laboratory, Industrial Technology Research Institute (ITRI), Hsinchu. In September 1997, he joined Metrodyne Microsystem Corporation, Hsinchu, and established the microelectromechanical systems (MEMS), Device Division, where he was the Manager between 1997 and 2000. He also established the first Micromachining Laboratory for commercial purpose in Taiwan. During 1998, he was an Adjunct assistant Professor in the Department of Electro-Physics, National Chiao Tung University. From 2001 to 2005, he was the Adjunct Assistant Professor in the Institute of Precision Engineering, National Chung Hsing University. In August 2001, he cofounded the Asia Pacific Microsystems (APM), Inc., Hsinchu, where he was the Vice President (VP) of R\&D, then became the VP of the Optical Communication Business Unit and also a special Assistant of the Chief Executive Officer (CEO) in charge of international business and technical marketing for MEMS foundry service till the end of 2005. He is currently an Assistant Professor with the Department of Electrical and Computer Engineering, National University of Singapore, Singapore. $\mathrm{He}$ is also a Senior Member of the Technical Staff, Institute of Microelectronics (IME), Agency for Science, Technology and Research (A*STAR), Singapore. He has authored or coauthored more than 110 international conference papers and extended abstracts, 60 peer-reviewed international journal articles, and holds eight U.S. patents in MEMS and nanotechnology field.

Dr. Lee is a member of the Materials Research Society (MRS) and the Institute of Electrical Engineers (IEE) Japan.

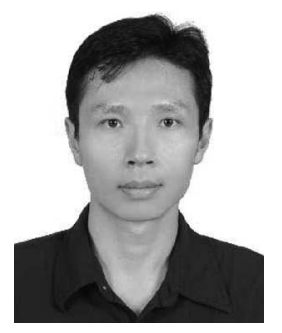

Fu-Li Hsiao received the B.S. degree from the Department of Physics, National Changhua University of Education, Changhua, Taiwan, in 2002, and the Ph.D. degree from the Department of Optics and Photonics, National Central University, Jhongli, Taiwan, and the Franche-Comté Electronique Mécanique Thermique et Optique-Sciences et Technologies (FEMTO-ST), University of FrancheComte, Besançon, France, in 2008.

$\mathrm{He}$ is currently a Research Fellow with the Department of Electrical and Computer Engineering, National University of Singapore, Singapore. His current research interests in clude photonic crystals, optical microelectromechanical systems (MEMS), and phononic crystals.

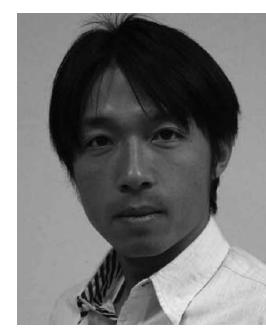

Takeshi Kobayashi received the B.S., M.S., and $\mathrm{Ph} . \mathrm{D}$. degrees in materials science from the University of Tokyo, Tokyo, Japan, in 1997, 1999, and 2002, respectively.

He is currently a Researcher at the National Institute of Advance Industrial Science and Technology (AIST), Tsukuba, Japan. His current research interests include piezoelectric microelectromechanical systems (MEMS) devices and their application to wireless sensor network. He has authored or coauthored 30 international journal papers and 21 conference proceedings. Two of his recent publications have been selected as highlighted papers of the Journal of Micromechanics and Microengineering in 2007 and 2008.

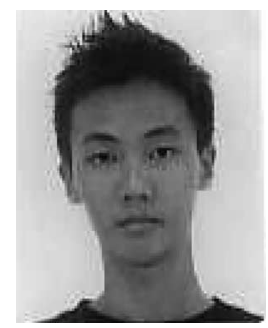

Kah How Koh received the B.S. degree in 2009 from the Department of Electrical and Computer Engineering, National University of Singapore, Singapore, where he is currently working toward the Ph.D. degree.

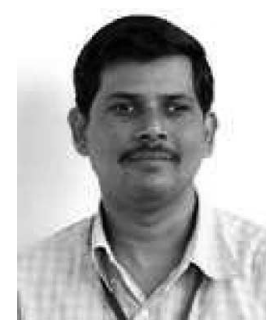

P. V. Ramana received the Master's degree in applied optics in 1984.

He was with the Military Research Institute, Ministry of Defence, Government of India, and was engaged in imaging and nonimaging optical guidance for missile systems. He was also with the R\&D Departments of Philips and Agilent Technologies, and worked in the field of optoelectronics. Since November 2001, he has been a Member of the Technical Staff (MTS), Institute of Microelectronics (IME), Agency for Science, Technology and Research (A*STAR), Singapore. He has more than 23 years of experience in R\&D of optoelectronics in research institutes and multinational companies. His current research interests include optical interconnects, optical imaging, illumination optics, and optical component packaging. He has authored or coauthored more than 50 research publications and ten invention disclosures in optics-related products. 


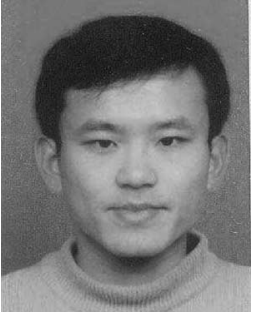

Wenfeng Xiang received the B.Sc. degree from the Department of Physics, Shandong Normal University, Jinan, China, in 2000, and the Ph.D. degree in materials science and engineering from the Institute of Physics, Chinese Academy of Sciences, Beijing, China, in 2005.

From August 2005 to August 2006, he was a Postdoctoral Fellow in the Department of Materials Science and Engineering, Gwangju Institute of Science and Technology, Gwangju, Korea. From September 2006 to December 2007, he was a Research Fellow in the School of Electrical and Electronic Engineering, Nanyang Technologica University. He is currently a Research Fellow with the Department of Electrical and Computer Engineering, National University of Singapore, Singapore. His current research interests include optical microelectromechanical systems (MEMS), nanophotonics sensor, and optical communications.

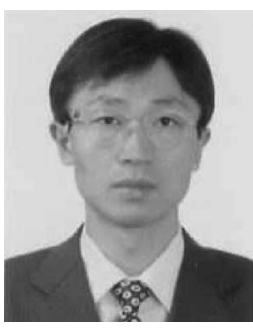

Bin Yang received the B.S. degree in materials science from Anhui University of Technology and Science, Huainan, China, the M.S. degree in materials science from Jilin University, Changchun, China, in 2003, and the Ph.D. degree in electronic science and technology from Shanghai Jiao Tong University, Shanghai, China, in 2006.

From August 2006 to October 2007, he was a Research Fellow in the Department of Mechanical Engineering, National University of Singapore, Singapore. He is currently a Senior Search Engineer at the Institute of Microelectronics (IME), Agency for Science, Technology and Research (A*STAR), Singapore. His current research interests include optical microelectromechanical systems (MEMS), transducers, piezoelectric actuators, and sensors.
Chee Wei Tan, photograph and biography not available at the time of publication.

D. Pinjala (M'01), photograph and biography not available at the time of publication. 